\title{
Small- and Wide-Angle X-Ray Scattering (SAXS/WAXS) with Temperature-Controlled Stages Makes Phase Identification Faster than Ever
}

\author{
Han $\mathrm{Wu}^{1}$ and Duncan Stacey ${ }^{2 *}$ \\ ${ }^{1}$ Centre for Nature Inspired Engineering, University College London, UK \\ ${ }^{2}$ Linkam Scientific Instruments, Surrey, UK \\ *duncanstacey@linkam.co.uk
}

\begin{abstract}
X-ray diffraction (XRD) is a fingerprint technique for the analysis of atomic and molecular structures of crystalline materials, from polymers and plastics, through to structural composites and biomaterials. These all have crystallographic phases in the nanostructure, which greatly influence the macro properties of the material-from insulin and hemoglobin to semiconductors and solar cells. Here, we look at how XRD analysis using a small- and wideangle X-ray scattering (SAXS/WAXS) system under full vacuum brings the possibility of crystallographic sample characterization, with temperature and environmental control, direct to the laboratory, and how this improves the workflow for phase identification.
\end{abstract}

Keywords: X-ray diffraction, small- and wide- angle X-ray scattering, crystal, temperature control, in-house

\section{Introduction}

X-ray diffraction (XRD) encompasses various experimental techniques that work by irradiating the sample with incident X-rays in order to measure the elastic scattering angle and intensity of the X-rays leaving the sample material. The diffraction pattern and intensity can correlate directly with specific crystalline components in the sample, giving a picture of the crystal phase, as well as details on defects and deformities within the material that deviate from the perfect crystal structure. Characterization using temperature-controlled XRD is a useful analytical tool for solids, liquids, and powder samples across many research applications, including solar cell research, drug discovery, and life sciences (Figure 1).

There are various forms of X-ray scattering techniques, including small- and wide-angle X-ray scattering (SAXS and WAXS), which differ by measuring the scattered X-rays at small $\left(2 \theta=0-5^{\circ}\right)$ or wide angles $\left(2 \theta>5^{\circ}\right)$, respectively. While SAXS and WAXS work widely with powder or liquid samples, grazing incidence (GI) SAXS and WAXS is often applied for thin film samples that can be irradiated at a shallow incident angle close to the critical angle $\left(0.1^{\circ}\right.$ to $\left.1^{\circ}\right)$.

Synchrotron facilities, such as Diamond in the UK, offer high-powered X-ray beamlines capable of performing XRD analysis on a wide range of samples. These national centers often host multiple beamlines with specific modifications for SAXS or WAXS, and traditionally this has been the only route for researchers to access this type of analysis. More recently, in-house XRD and SAXS/WAXS devices, which scale down the beamline to enable the equipment to fit within a standard laboratory, have become available. These bring quicker and easier access to crystallographic characterization, with more control over sample conditions for environmental analysis, such as temperature, humidity, and gas flow.

One such in-house SAXS/WAXS system is housed at the Centre for Nature-Inspired Engineering (CNIE), Department of
Chemical Engineering, at University College London. Established in 2014, the CNIE research facility supports multi-disciplinary research across the University, and access is available for academic and industrial collaborators and users. Access to the facility can be obtained by contacting Dr. Han Wu via their website (https://www.ucl.ac.uk/nature-inspired-engineering/facilities).

Users of the facility can access a range of instruments worth over $£ 6$ million to characterize their samples, including: liquid TEM, a nano 3D printer, a dynamic light scattering (DLS) system, a differential scanning calorimeter (DSC), and thermal gas thermogravimetric analysis (TGA) and TGA mass spectrometry (TGA/MS) instruments. Among its state-of-the-art instruments, the facility has a unique in-house small/wide-angle X-ray scattering (SAXS/WAXS) instrument, the Ganesha 300XL. Used independently, and in tandem with the Ganesha 300XL and other instruments, are several temperature-controlled stages, including the Linkam capillary X-ray stage and the HFSX350-CAP stage, which has a capillary tube through the heating block to enable observation of the temperature-dependent behavior of food and pharmaceuticals, batteries, fuel cells, catalysis, and architecture materials; and the optical Linkam DSC450, which provides enthalpy data (Figure 2). The temperature-controlled DSC450 enables liquid nitrogen temperatures without the need for large liquid nitrogen dewars when performing temperature-controlled experiments. Additional temperature-controlled stages include the HFSX350-CAP, which allows sample preparation inside a small capillary, and thus carefully controlled environmental condition at high vacuum inside an XRD chamber.

\section{In-House SAXS/WAXS for Rapid Phase Identification}

$\mathrm{X}$-ray scattering is a well-established technique for the characterization of polymers, pharmaceuticals, nanoparticles, and biological materials [1-3]. WAXS provides structural information at the atomic scale down to $0.1 \mathrm{~nm}$, similar to traditional XRD. SAXS provides information on complex molecules and materials such as polymers, colloids, and porous materials, up to $500 \mathrm{~nm}$ in size. Typically, SAXS/WAXS is used for small-angle scattering studies. However, with the CNIE equipment's whole-vacuum setup, wide-angle X-ray scattering can offer rapid data collection with very precise temperature control and easy sampling. There is also an important energysaving benefit because cryo temperatures can be obtained with just 1 liter of liquid nitrogen. The whole-vacuum SAXS/WAXS system that was developed by SAXSLAB can be used to evaluate multi-scale structures, such as atomic structure $(0.2 \mathrm{~nm}$ to $1 \mathrm{~nm})$ and molecular structure ( $1 \mathrm{~nm}$ to $100 \mathrm{~nm}$ ). It can also be used 


\section{More Wavelengths. More X-Gite.}

\section{Microscoppy 2021 Innovation Award}

- Full spectral coverage from Fura-2 to IR800

- 9-channel wavelength selection

- LaserLED Hybrid Drive ${ }^{\circledR}$ with 4-position motorized filter changer

- Pre-installed clean-up filters

- Whisper quiet operation

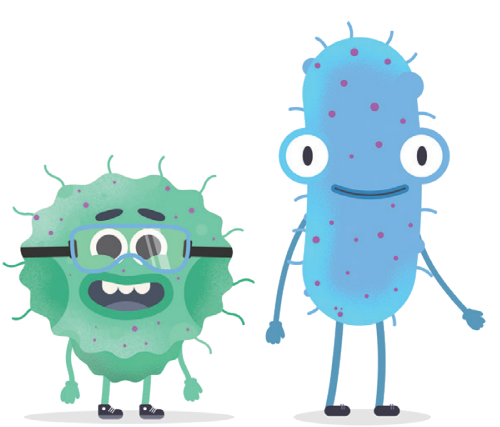

Engage with us at: Neuroscience 2021

Booth \#601

Chicago, IL

November 14-16, 2021 


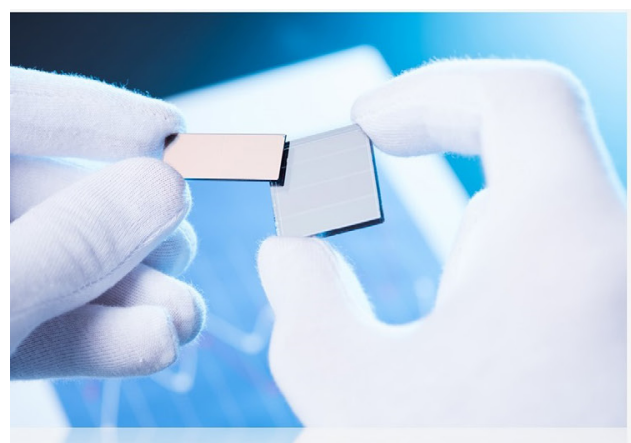

Photovoltaics

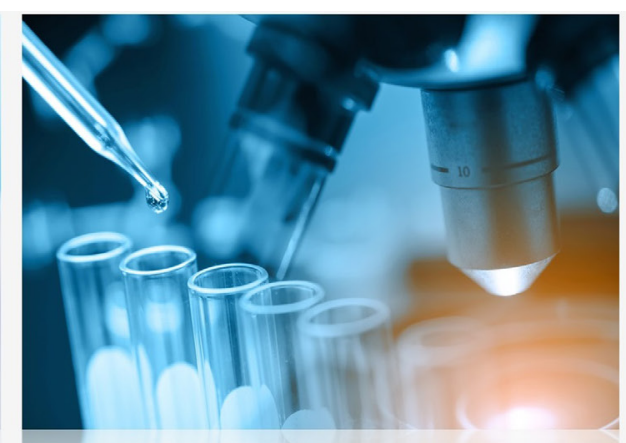

Pharmaceuticals

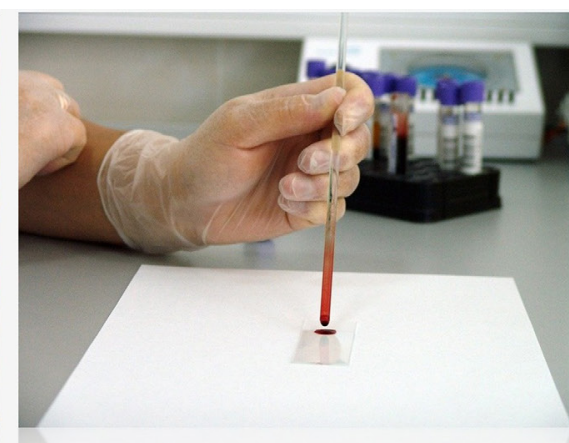

Biomaterials

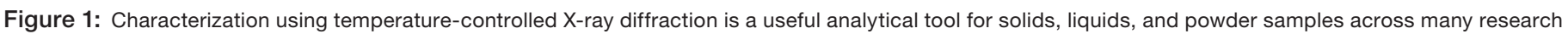
applications, including solar cell research, drug discovery, and life sciences. Stock images used with permission from Shutterstock, iStock, and Pixabay.

to study materials with different forms, including solids (for example, powders, pastes, or thin films), liquids, liquid-crystals, and gels (with ordered and disordered structures). When coupled with a moving detector and automated alignment/ calibration routine, phase identification is fast and accurate.

The Ganesha 300XL provides extensive automation, a large sample chamber, and versatile sample area to provide a highly customized instrument for temperature-dependent analysis between $-175^{\circ} \mathrm{C}$ and $350^{\circ} \mathrm{C}$, including analysis of solutions in refillable capillaries from $4^{\circ} \mathrm{C}$ to $80^{\circ} \mathrm{C}$, and time-resolved micro-fluidics monitoring in air. Additionally, the instrument can be used for grazing angle experiments (GISAXS/GIWAXS), which is useful for thin films such as photovoltaic devices. Key applications of the Ganesha 300XL include nano-particle size, shape, distribution, and polydispersity analyses; threedimensional protein molecule structure analyses (folding and unfolding); identification of molecular assembly or disassembly;

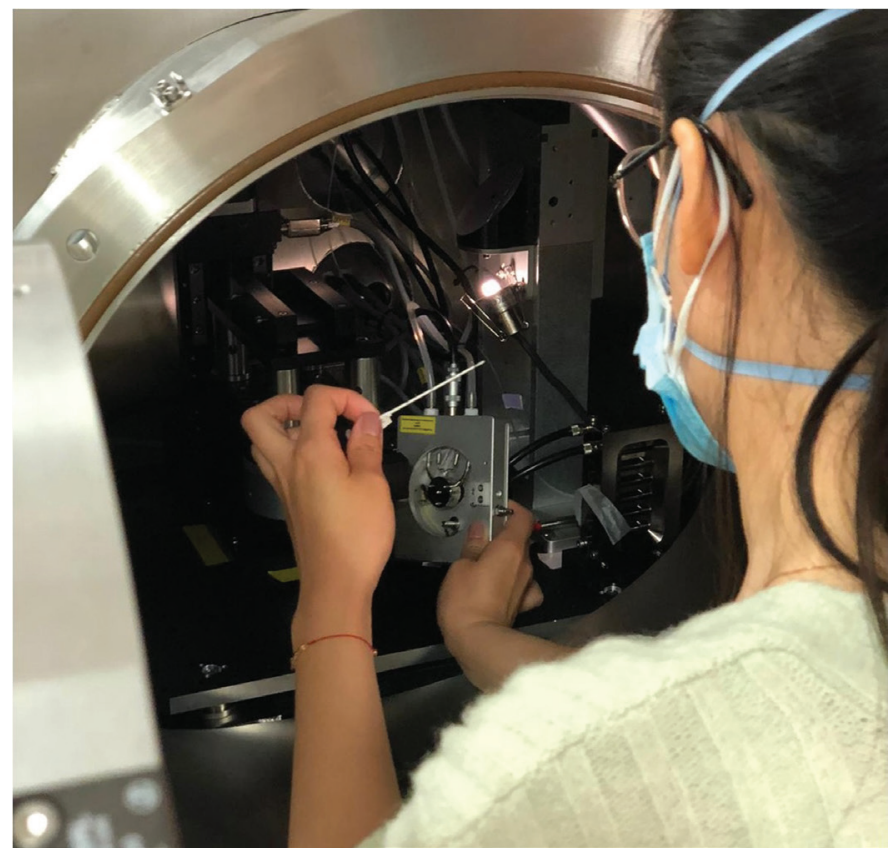

Figure 2: CNIE researcher fitting the HFSX350-CAP inside Ganesha's SAXS/ WAXS chamber. and colloids, metals, cement, oil, polymers, plastics, foods, and pharmaceuticals. The setup at CNIE (Figure 3) can be widely applied to detect new polymorphs and phase changes in both solid and liquid samples. In order to study the temperaturedependent properties of these materials, such as phase changes at high or cryo temperatures, the Ganesha can be equipped with Linkam capillary or GI stages to enable in situ crystallographic measurements as the sample conditions change.

\section{Speed is of the Essence}

Using a Linkam HFSX350-CAP temperature-control capillary stage, it is possible at the CNIE to conduct timeresolved SAXS/WAXS studies of the phase behavior of pharmaceutical compounds under controlled heating/cooling conditions $\left(-195^{\circ} \mathrm{C}\right.$ to $\left.350^{\circ} \mathrm{C}\right)$ at a remarkable rate of 1 second per scan, while achieving distinct and clear scattering data, as shown in Figure 4. In traditional XRD, 30 minutes to an hour is required to collect a pattern. Using the Linkam stage it is possible to heat and cool a sample rapidly and collect the pattern in just 1 or 2 seconds.

In prior research at the University of Sheffield in 2010 [4], Han Wu, the current CNIE Research Facility Manager, studied pyroglutamic acid $(\mathrm{P})$, which occurs naturally in fruits, some plant foods, dairy, and fermented products, such as soya sauce, and can be produced by thermal dehydration and cyclization of glutamic acid $[5,6]$. The brain-boosting effects of $\mathrm{P}$ were discovered in 1984 [7], and it plays an important role in the preservation and activity of key neurotransmitters. P esters can be used as dermal penetration enhancers for therapeutic agents having poor skin permeation [8] or hair growth agents [9]. In chemical synthesis, it has been used as a versatile chiral building block in asymmetric synthesis of alkaloids [10], pharmaceuticals, and many other natural products.

$\mathrm{Han} \mathrm{Wu}$ and her colleagues discovered three polymorphs for P: $\alpha^{\prime}$ (low-temperature polymorph); $\alpha$ (ambient temperature polymorph); $\beta$ (high-temperature polymorph), and two phase changes $\left(\alpha / \beta\right.$ and $\left.\alpha^{\prime} / \alpha\right)$. The $\beta$ and $\alpha^{\prime}$ forms had not been recognized prior to this work. They found that the change from $\alpha^{3} / \alpha$ happens slowly over a range of very low temperatures (starting at $-140^{\circ} \mathrm{C}$ ) and appeared to be Martensitic in character, in which strain at the $\alpha / \alpha$ interface in partially transformed crystals caused the transformation to occur in bursts with increased undercooling. 

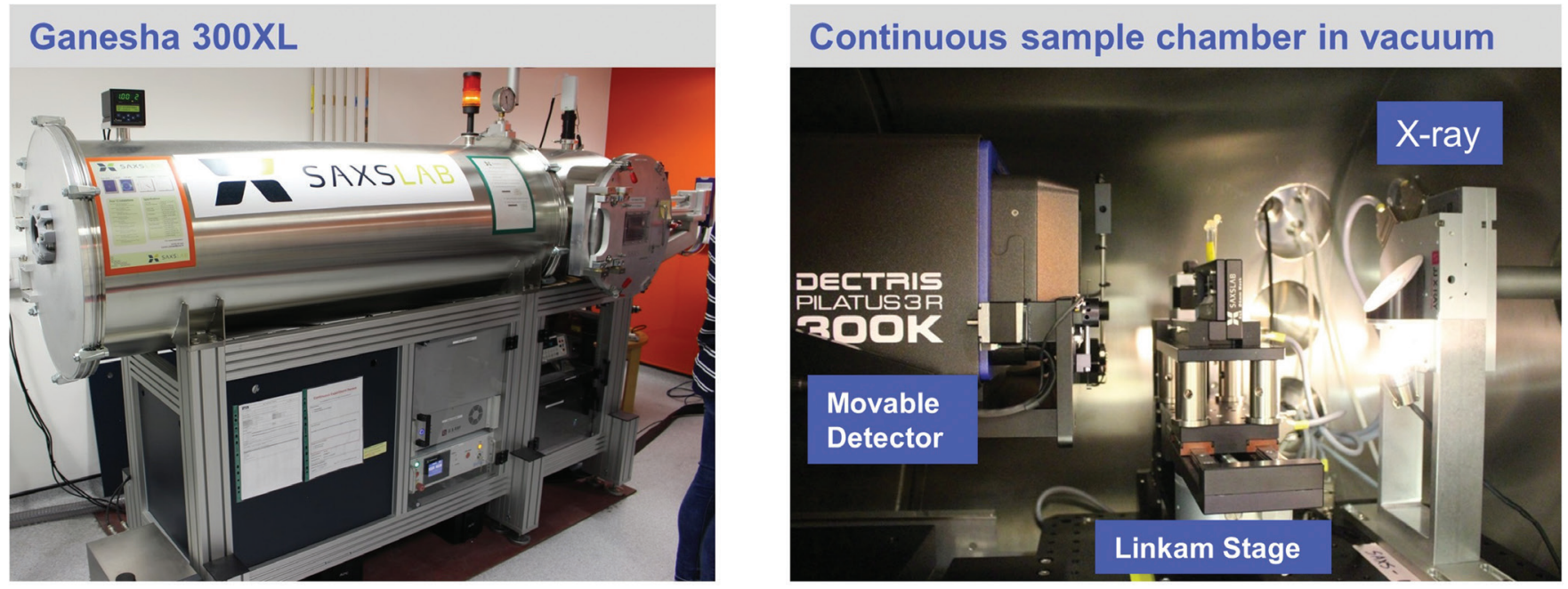

Different sample holders/stages for capillaries, temperature control, etc.
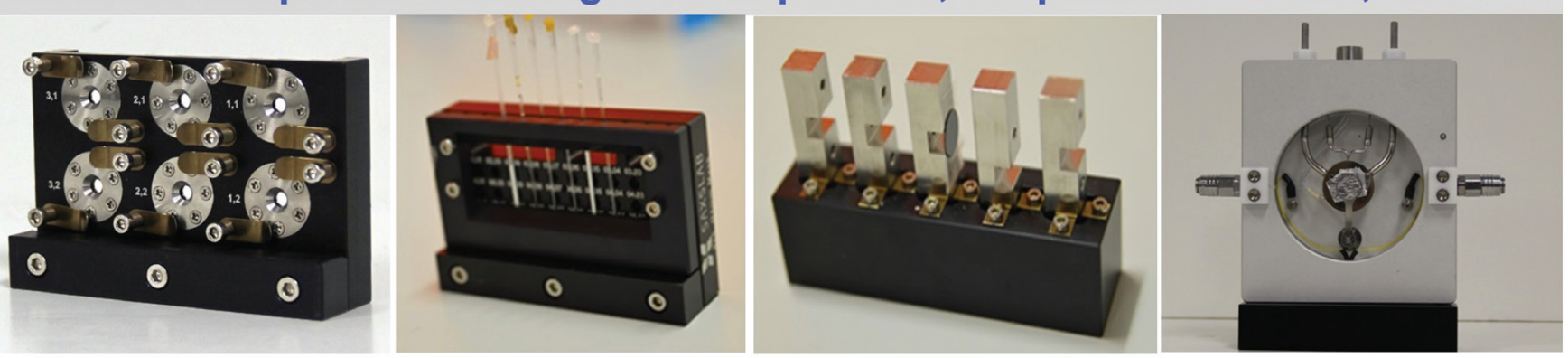

Figure 3: Top: Whole-vacuum SAXS/WAXS system at CNIE, including the Ganesha 300XL and moving detector. Bottom, left to right: Sample stages compatible with Ganesha. Sandwich cells, capillaries, and folded foil envelope can be used with the Linkam HFSX350-CAP (upper right) and enable temperature control across a wide range of samples.

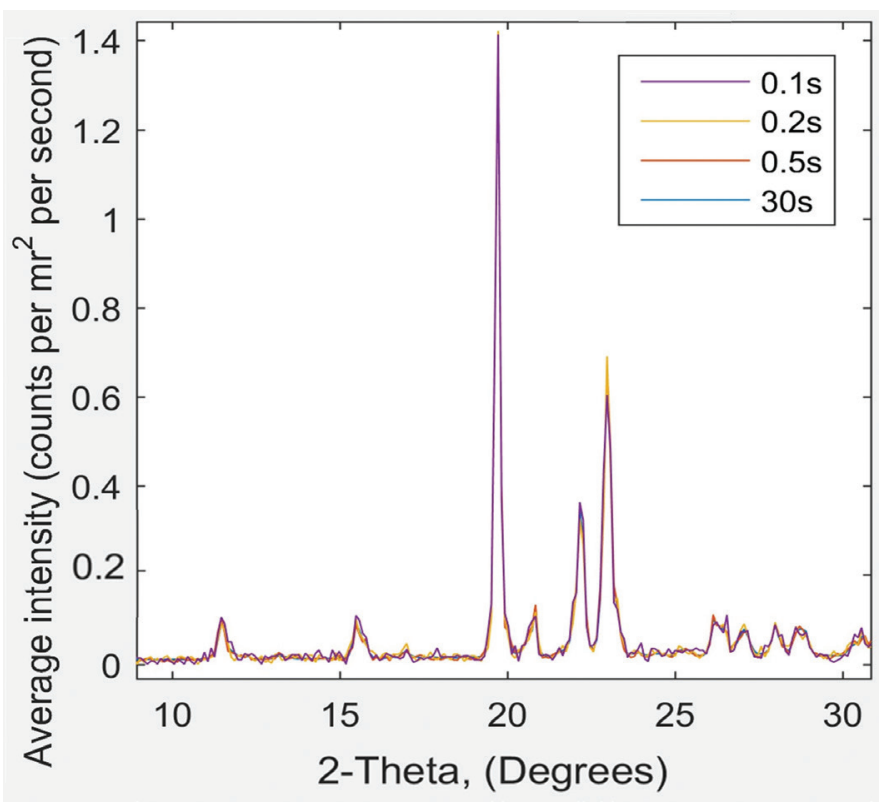

Figure 4: XRD scattering data from Ganesha 300XL for pyroglutamic acid at room temperature: $2 \theta$ as a function of scattering intensity for data captured over $0.1,0.2,0.5$, and 30 s runs. The four characteristic pyroglutamic acid peaks show no difference between the $0.1 \mathrm{~s}$ and $30 \mathrm{~s}$ analyses. Note that some $30 \mathrm{~s}$ curves are not visible as they are hidden behind the other curves.
The $\beta / \alpha$ transition was single crystal to single crystal, representing a rapid phase transformation that could be significantly suppressed at fast cooling rates. Although there was little change in overall volume of crystals on passing

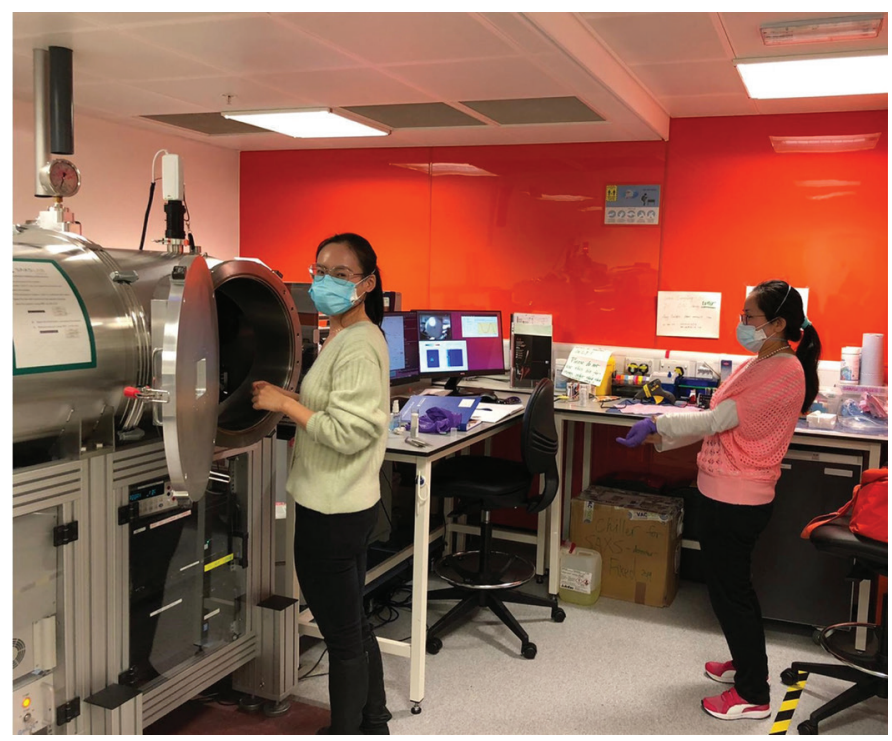

Figure 5: Han (right) and a colleague working in the CNIE SAXS/WAXS laboratory. 
through the $\alpha / \beta$ transition, there were significant changes of $\sim 1 \%$ in the individual unit cell parameters-and therefore in crystal dimensions-on passing through the transition in either direction. This had the effect of causing crystals to jump off a solid surface, such as a microscope slide, on passing through the transition.

$\mathrm{Han} \mathrm{Wu}$ recently reran the analysis of $\mathrm{P}$ and its phase transitions, which in her prior work from 2010 took 10 hours (with 200L liquid nitrogen dewar). Using the Ganesha 300XL and the Linkam stage (Figure 5) with the 1L liquid nitrogen dewar, she was able to complete the entire range of phase transformations from $80^{\circ} \mathrm{C}$ to $-170^{\circ} \mathrm{C}$ in a couple of hours and collect each data set in just 2 seconds using WAXS (Figure 6). She was also able to actually see the crystals "jumping" using the open DSC system and microscope (Figure 7) and correlate these images with the DSC curve (Figure 8).

The temperature-controlled SAXS/WAXS system at CNIE has shown it is capable of excellent sample characterization performance, with several key advantages: 1) time: quickest scan in 1 second, which is powerful for phase identification and polymorph screening; 2) high signal-to-noise ratio: minimal scattering from air and windows due to whole-vacuum setup; 3 ) small-angle scattering from $0.004^{\circ}$ to $5^{\circ}$ in $2 \theta$ and wide-angle scattering up to $60^{\circ}$ in $2 \theta$; 4) temperature-dependent studies: accurate temperature control from as low as $-190^{\circ} \mathrm{C}$ up to $300^{\circ} \mathrm{C}$ using just $1 \mathrm{~L}$ of liquid nitrogen to achieve controllable heating/ cooling; 5) characterization of short-lived intermediate phases.

\section{Conclusions}

SAXS/WAXS has great potential for rapid polymorph screening when traditional XRD is not adequate and synchrotron access is not readily available. The CNIE system is much more than an SAXS, and in many application areas, it is superior to most lab XRD instruments in terms of time and energy saving, with comparable results. The incorporation of the Linkam stage enables collection of temperature-dependent measurements, making it possible to detect new polymorphs and phase changes in both solid and liquid samples.
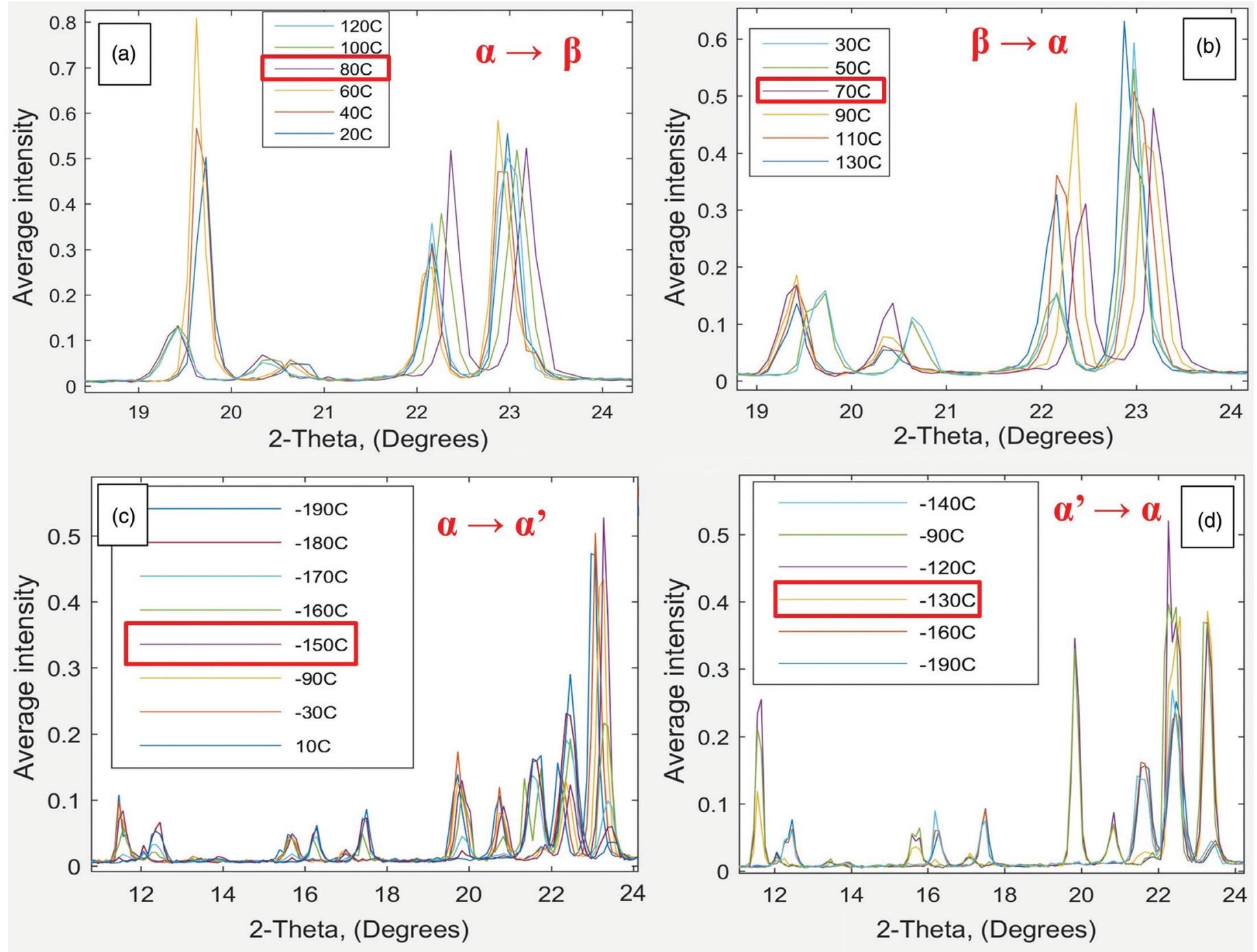

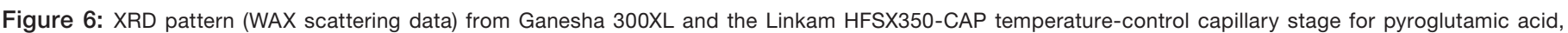

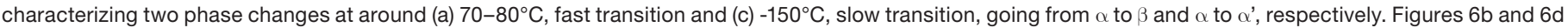
show the respective reverse transitions. Data collected at temperature intervals of $10^{\circ} \mathrm{C}$ using WAXS in 2 seconds. 

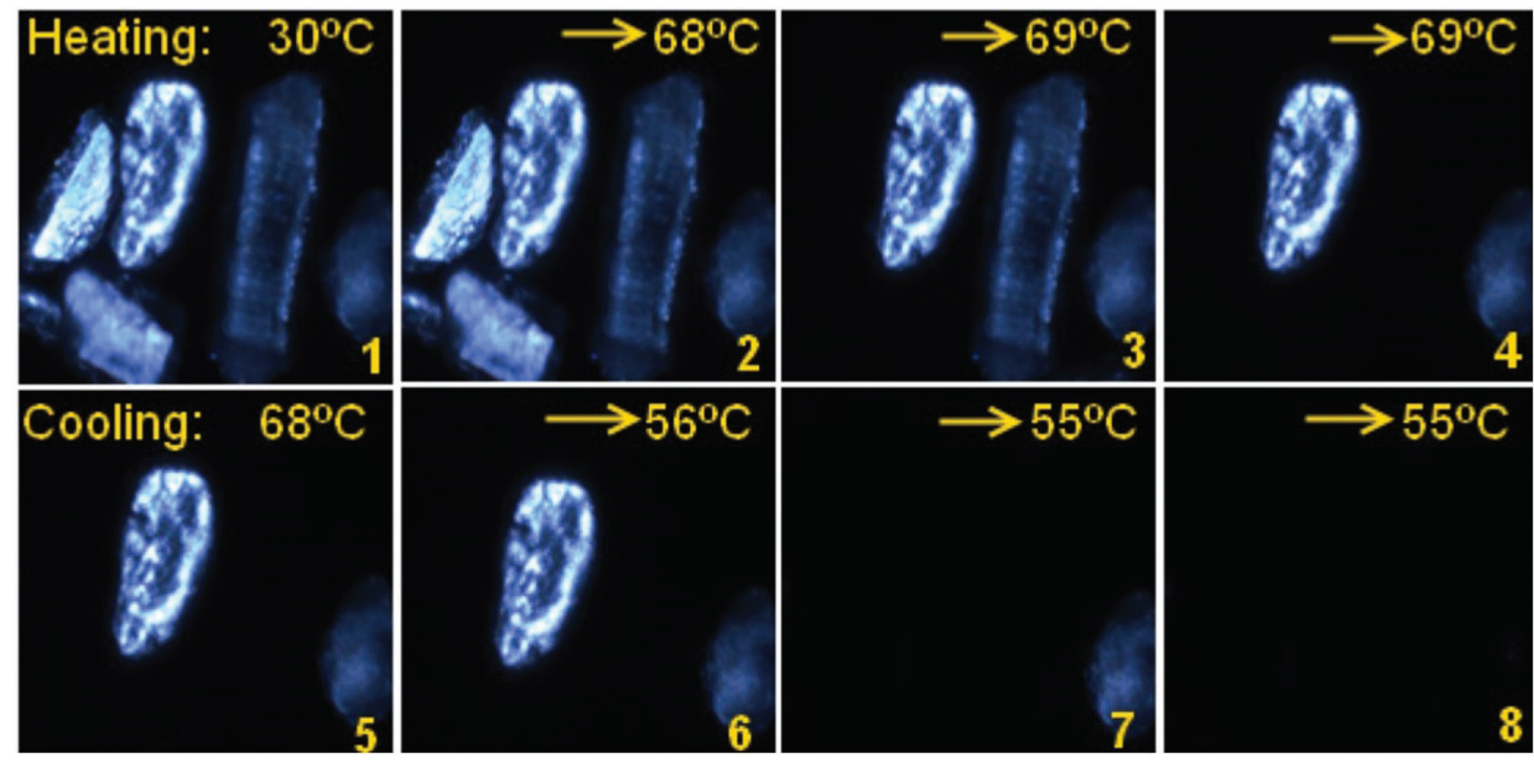

Figure 7: Hot-stage polarizing microscope pictures of L-P crystals at different temperatures on heating (1-4) and subsequent cooling (5-8); crystals move at $\sim 69^{\circ} \mathrm{C}$ on heating and $\sim 55^{\circ} \mathrm{C}$ on cooling. By hot-stage optical microscopy, significant changes to crystals of L-P were seen on both heating and cooling through the $\mathrm{R} T$ $\beta$ transition. Specifically, some crystals, especially larger ones, jumped around the glass slide holding the samples, as shown by the sequence of micrographs on heating (1-4) and subsequent cooling (5-8). Starting with six crystals in the field of view at $30^{\circ} \mathrm{C}$ and $68^{\circ} \mathrm{C}$, three of these disappeared on increasing the temperature to $69^{\circ} \mathrm{C}$, and a further one disappeared on holding at $69^{\circ} \mathrm{C}$. On subsequent cooling, one of the two remaining crystals jumped out of the field of vision at $55^{\circ} \mathrm{C}$, and the other one also disappeared on holding at $55^{\circ} \mathrm{C}$
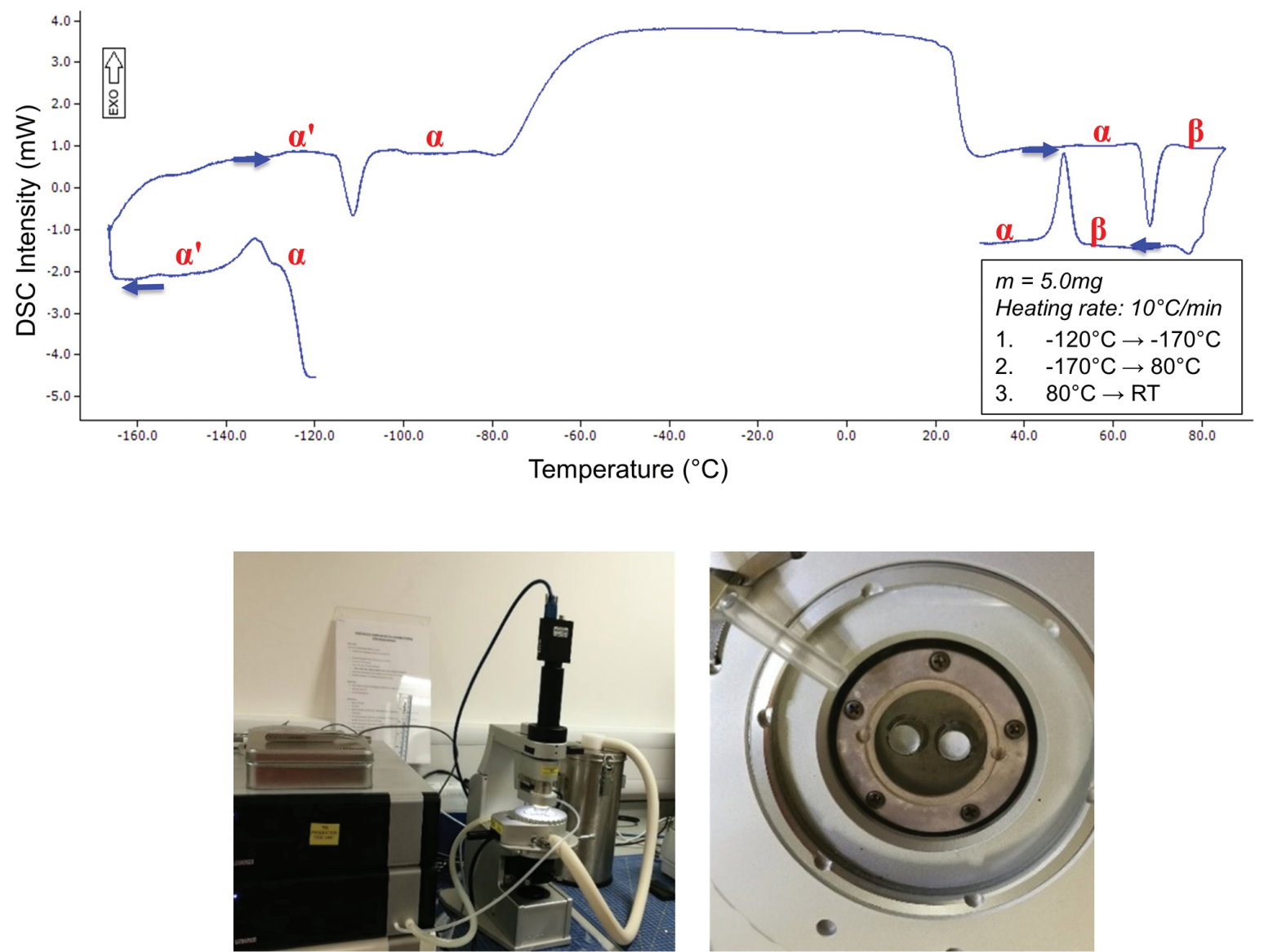

Figure 8: (Top) Corresponding DSC trace showing $\alpha$ to $\beta$ and $\alpha$ to $\alpha$ ' phase transitions for pyroglutamic acid. (Bottom) Standalone optical DSC450 setup and close-up of sample and reference crucibles with quartz windows enabling imaging. 
It is clear from the examples described here that the equipment at CNIE enables researchers to easily conduct crystallographic sample characterization, with temperature and environmental control, improving the workflow for phase identification across a wide variety of materials and applications.

\section{References}

[1] J Lipfert and S Doniach, Ann Rev Biophys Biomol Struct 36 (2007) https://doi.org/10.1146/annurev.biophys.36.040306 .132655 .

[2] B Chu and BS Hsiao, Chem Rev 101 (2001) https://doi .org/10.1021/cr9900376.

[3] T Li et al., Chem Rev 116 (2016) https://doi.org/10.1021/ acs.chemrev.5b00690.

[4] H Wu et al., Cryst Growth Des 10 (2010) https://doi .org/10.1021/cg100260f.

[5] CB Park et al., Appl Env Microbiol 67 (2001) https://doi .org/10.1128/AEM.67.8.3650-3654.2001.

[6] AF Beecham, J Am Chem Soc 76 (1954) https://doi .org/10.1021/ja01647a033.

[7] T Antonelli et al., Pharmacol Res Comm 16 (1984) https:// doi.org/10.1016/s0031-6989(84)80094-6.

[8] J Alexander and T Higushi, US Patent 5,066,648, filed 1990, issued 1991.

[9] WT Gibson and IR Scott, US Patent 5,158,955, filed 1991, issued 1992.

[10] JL Marco, J Heterocyclic Chem 23 (1986) https://doi. org/10.1002/jhet.5570230419.

\section{SEM Scintillators \& Light Guides}

Light Guide Recoating Services too!

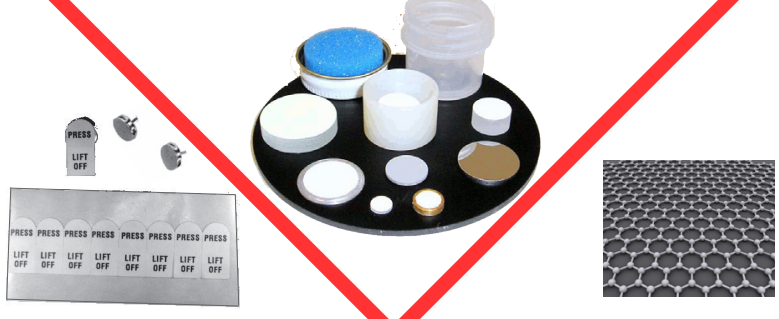

Conductive Adhesives, Introducing GRAPHENE Coatings \& Tabs!

The New Carbon!

Brush or Spray

the same product!

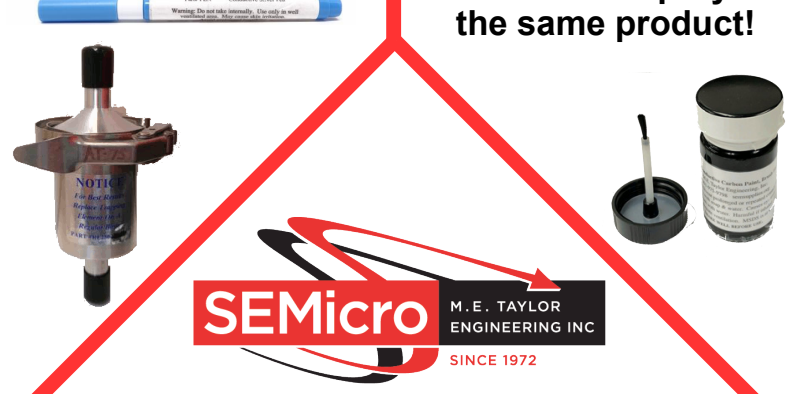

www.semsupplies.com • (301) 975-9798

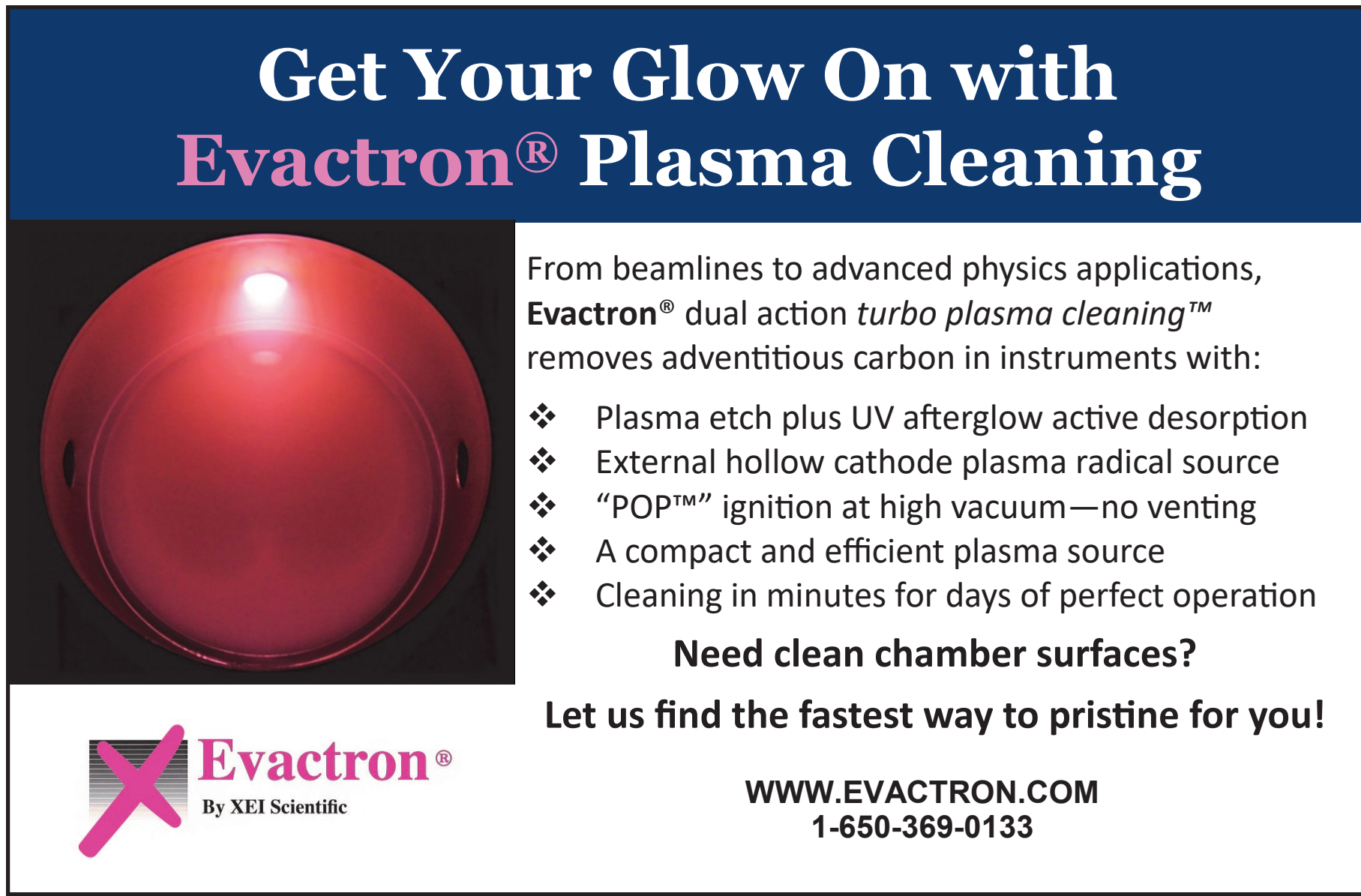




\section{D Mreromac}

\section{microPREPTM PRO}

High-Speed Sample Preparation

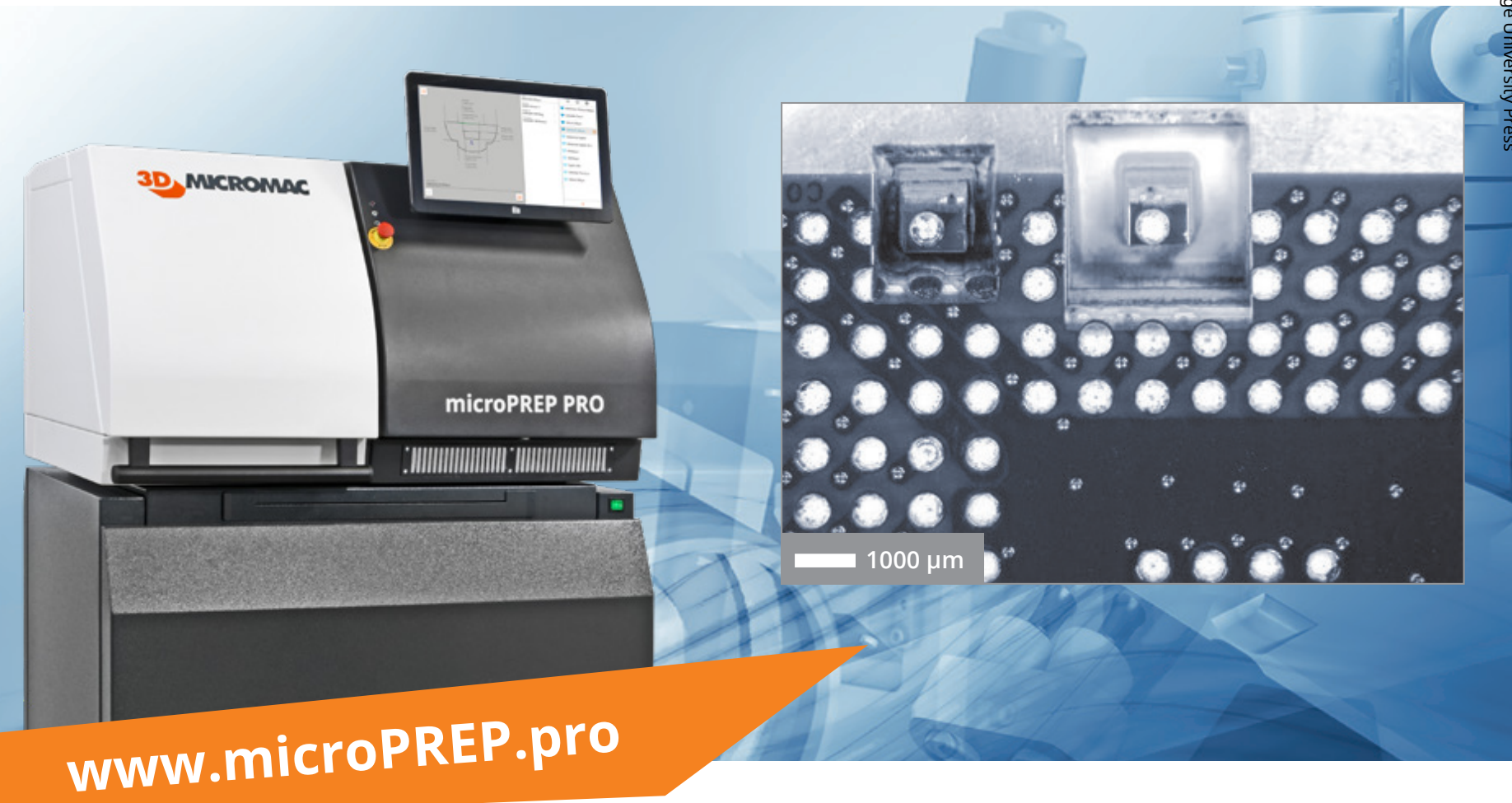

» Free-form laser-based sample preparation

» Larger-sized samples with micron-level precision

»Workflows for TEM, XRM, APT, and micromechanical testing

»Reduces time-to-sample and costs significantly

Contact us now!

Tel: $+4937140043-222$

sales@3d-micromac.com

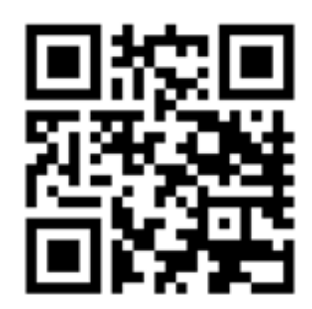

